\title{
Surgical Outcomes of Medial Rectus Recession and Lateral Rectus Resection for Large-angle Deviations of Acute Acquired Concomitant Esotropia
}

\author{
Wonki Kang, Won Jae Kim \\ Department of Ophthalmology, Yeungnam University Medical Center, Daegu, Korea
}

Purpose: This study aimed to evaluate the surgical outcomes of unilateral medial rectus recession and lateral rectus resection (R\&R) for patients with large-angle deviations of acute acquired concomitant esotropia (AACE) without a neurological disease. Further, we have also suggested the surgical dosage of R\&R for large-angle deviations of AACE.

Methods: This was a retrospective study of patients with AACE who underwent surgery and followed up after surgery for at least 6 months between September 2016 and March 2020. Among them, patients with ocular deviations of $\geq 30$ prism diopters (PD) who underwent unilateral R\&R were included.

Results: Seventeen patients with AACE were enrolled in this study (mean age, 23.5 years; four females). The mean amount of deviation was 40.00 PD at distance and near (range, 30-55 PD). Fifteen patients (88.2\%) had myopia. The mean interval between the initial visit and surgery was 7.7 months (range, 4-20 months). All included patients had resolved diplopia after the surgery. Most patients with decreased stereoacuity displayed stereoacuity recovery after surgical treatment. At the final visit (mean follow-up period, 12.8 months), the mean angle of esodeviation was 0.82 PD (range, 6-0 PD) at distance and 0.47 PD (range, 4-0 PD) near. No patient displayed over-correction or adduction limitations in the operated eye.

Conclusions: The unilateral R\&R procedure presented favorable surgical outcomes for patients with large-angle deviations of AACE. These results and proposed surgical dosage guidelines may be useful for surgeons in planning the surgical treatment of large-angle deviations of AACE.

Key Words: Diplopia, Esotropia, General surgery, Strabismus

Acute acquired concomitant esotropia (AACE) is a relatively rare condition that is characterized by the acute onset of esotropia with diplopia [1-5]. Ophthalmic and neurological evaluations, including neuroimaging, should be

Received: April 20, 2020 Final revision: January 31, 2021

Accepted: February 14, 2021

Corresponding Author: Won Jae Kim, MD. Department of Ophthalmology, Yeungnam University Medical Center, 170 Hyeonchung-ro, Nam-gu, Daegu 42415, Korea. Tel: 82-53-620-3440, Fax: 82-53-626-5936, E-mail: eyekwj@ynu.ac.kr conducted to rule out any associated intracranial pathology and guide treatment $[2,5]$. The treatment of esotropia and diplopia is the primary focus for patients with AACE without a neurological disease. Patients with large-angle deviations of AACE usually require surgical treatment for esotropia [5]. Previous studies have suggested that strabismus surgery for AACE can successfully restore good binocular function and improve diplopia in patients without underlying intracranial issues [4-9].

Bilateral medial rectus (MR) recession or unilateral medial rectus recession and lateral rectus resection $(R \& R)$ are 
the two most common procedures performed for the surgical treatment of esotropia. There is no evidence of superiority of one surgical procedure [6]. The surgical methods may be different according to the surgeon's preference. Initially, we performed a bilateral MR recession for the treatment of patients with moderate angle deviations of AACE based on the traditional surgical dosage. When we reviewed the surgical outcomes in these patients, we found that some patients had under-correction. Previous study has rarely evaluated the surgical outcomes of unilateral $\mathrm{R} \& \mathrm{R}$ for large-angle deviations of AACE and proposed surgical dosage guidelines. Therefore, we evaluated the surgical outcomes of R\&R for patients with large-angle deviations of AACE without a neurological disease and proposed surgical dosage guidelines.

\section{Materials and Methods}

This was a retrospective study in which patients with AACE who underwent surgery and followed up after surgery for at least 6 months between September 2016 and March 2020 were enrolled. AACE was defined as a sudden onset of concomitant (deviation in all directions of gaze differing by $\leq 5$ prism diopters [PD]) non-accommodative esotropia with diplopia. Among these patients, those with an ocular deviation of $\geq 30$ PD who underwent unilateral $R \& R$ procedures were included. Patients who were found to have any neurological disease associated with esotropia were excluded from this study. Patients with a prior history of strabismus surgery, any cause of fusion interruption, or a history of systemic disease associated with esotropia, were excluded. This study was performed in accordance with the tenets of the Declaration of Helsinki and was approved by the institutional review board of Yeungnam University Hospital (2020-03-107). Informed consent was waived by the board because of the retrospective nature of this study and the use of anonymized patient data.

\section{Clinical evaluation of patients with AACE}

All patients underwent a full ophthalmologic examination during their initial visit. This included visual acuity and ocular alignment status tests, slit-lamp biomicroscopy, refraction and fundus examinations, and a stereoacuity test. The angle of deviation was measured using an alter- nate prism cover test at $6-\mathrm{m}$ and $33-\mathrm{cm}$ fixation, both preoperatively and postoperatively. A positive number represented esodeviation, while a negative number represented exodeviation. Additionally, duction limitations were evaluated. Stereoacuity measurements were performed using the Lang I (Lang-Stereotest AG, Küsnacht, Switzerland) and Stereo Fly Stereotest (Stereo Optical Co., Chicago, IL, USA). Neurological workups, including magnetic resonance imaging of the brain with orbit, were performed to exclude neurological causes of esotropia in all patients. Serologic examinations, including a thyroid function test and anti-acetylcholine receptor antibody test, were conducted to evaluate the cause of esotropia. Surgery was performed when the amount of esodeviation was confirmed to be consistent over a follow-up period of at least 4 months. Each patient attended at least two preoperative examinations during the follow-up period. All surgeries were performed under general anesthesia by a single surgeon (WJK). The forced duction test was performed in all patients under general anesthesia. The conventional $R \& R$ procedure for esotropia was performed in all patients. The fornix incision was used in all patients except 3 adult patients. The operated muscles were reattached directly to the sclera with 6-0 Vicryl sutures in all patients. All patients routinely used Tobradex (tobramycin/dexamethasone) eye drops four times daily and Effexin (ofloxacin) eye ointment once daily for 1 week, postoperatively. Follow-up visits were conducted at 1 week, 1 month, and 3 months postoperatively, and every 6 months after that.

\section{Statistical analysis}

Continuous data are presented as a mean \pm standard deviation, and categorical data are presented as counts and percentages. Data were analyzed using IBM SPSS Statistics ver. 20.0 (IBM Corp., Armonk, NY, USA).

\section{Results}

\section{Basic characteristics of patients with AACE}

A total of 38 patients with AACE underwent surgical treatment during the study period. Among these, 18 patients underwent unilateral R\&R and 20 patients underwent bilateral MR recession for the treatment of AACE. 
Table 1. Basic characteristics of included patients with acute acquired concomitant esotropia $(\mathrm{n}=17)$

\begin{tabular}{|c|c|}
\hline Characteristics & Value \\
\hline Mean age at first visit (range) (yr) & $23.5(8-62)$ \\
\hline Mean duration of esodeviation (range) (mon) & $9.9(1-30)$ \\
\hline Sex (male : female) & $13: 4$ \\
\hline \multicolumn{2}{|l|}{ Spherical equivalent refractive errors (range) (D) } \\
\hline Right eye & $-3.60 \pm 2.73(-8.50$ to +0.50$)$ \\
\hline Left eye & $-3.44 \pm 2.57(-8.75$ to +0.50$)$ \\
\hline Mean interval from first visit to surgery (range) (mon) & $7.7(4-20)$ \\
\hline Mean age at surgery (range) (yr) & $24.2(9-62)$ \\
\hline \multicolumn{2}{|l|}{ Preoperative angle of deviation (range) (PD) } \\
\hline Distant & $40.00 \pm 7.50(30-55)$ \\
\hline Near & $40.00 \pm 7.70(30-55)$ \\
\hline \multicolumn{2}{|l|}{ Distribution of preoperative angle of deviation (PD) } \\
\hline$<40$ & 7 \\
\hline $40-50$ & 9 \\
\hline$>50$ & 1 \\
\hline Operated eye (right : left) & $7: 10$ \\
\hline \multicolumn{2}{|l|}{ Preoperative results of stereotest } \\
\hline Lang I test (failed, \%) & $9 / 16(56.3)$ \\
\hline Stereo Fly Stereotest (>100 arcsec, \%) & $11 / 16(68.8)$ \\
\hline
\end{tabular}

$\mathrm{D}=$ diopters; $\mathrm{PD}=$ prism diopters; arcsec $=$ arcsecond.

Table 2. Surgical dosage of unilateral R\&R for patients with large-angle deviations of acute acquired concomitant esotropia

\begin{tabular}{ccc}
\hline \multirow{2}{*}{ Prism diopters } & \multicolumn{2}{c}{ R\&R } \\
\cline { 2 - 3 } & $\begin{array}{c}\text { Recession amounts } \\
\text { of MR }\end{array}$ & $\begin{array}{c}\text { Resection amounts } \\
\text { of LR }\end{array}$ \\
\hline 30 & 5.0 & 4.0 \\
35 & 5.0 & 5.0 \\
40 & 6.0 & 6.0 \\
45 & 6.0 & 7.0 \\
50 & 6.0 & 8.5 \\
55 & 6.5 & 9.5 \\
\hline
\end{tabular}

$\mathrm{R} \& \mathrm{R}=$ medial rectus recession and lateral rectus resection; $\mathrm{MR}$ $=$ medial rectus muscle; $\mathrm{LR}=$ lateral rectus muscle.

One patient who underwent unilateral R\&R was lost to follow-up. Therefore, 17 patients with AACE were enrolled in this study.

The basic characteristics of the included patients are shown in Table 1 . The mean age at first visit was 23.5 years (range, 8-62 years). This study included nine pediatric patients $(\leq 18$ years old) and eight adult patients. The mean amount of deviation was $40.00 \pm 7.50 \mathrm{PD}$ at a distance and $40.00 \pm 7.70$ PD near. The angle of ocular deviation was nearly equivalent for distant and near fixation. Fifteen of the 17 patients $(88.2 \%)$ had myopia. Two patients had mild hyperopia. The R\&R procedure was performed for the largest angle of ocular deviation ever measured based on the surgeon's surgical dosage (Table 2). Three patients showed mild restriction on abduction at the forced duction test under general anesthesia.

\section{Surgical outcomes of R\&R for large-angle deviations of AACE}

The mean interval between the initial visit and surgery was 7.7 months (range, 4-20 months). The mean immediate postoperative angle of esodeviation was 0.24 PD (range, 2-0 PD) at distance and 0.00 PD (range, -2-0 PD) 
near. All included patients had improved diplopia after the surgery. Nine patients who failed the Lang I test before surgery passed the test after surgery. These patients also showed improvement in stereoacuity using the Stereo Fly stereotest after surgery. The mean postoperative follow-up period was 12.8 months (range, 6-23 months). At the final visit, the mean angle of esodeviation was $0.82 \mathrm{PD}$ (range, 6-0 PD) at distance and $0.47 \mathrm{PD}$ (range, 4-0 PD) near. No patient showed overcorrection or adduction limitations in the operated eye at the final visit.

\section{Discussion}

The unilateral R\&R procedure presented favorable surgical outcomes for patients with large-angle deviations of AACE. All patients showed improvement in diplopia. Most patients with decreased stereoacuity showed improvement in stereoacuity after surgical treatment. Our results may be useful for surgeons in planning the surgical treatment of large-angle deviations of AACE.

AACE is a rare form of esotropia that occurs in older children and adults and is characterized by the acute onset of esotropia with diplopia [2-5]. AACE has been traditionally divided into three distinct types. Type I (Swan type) occurs following the disruption of fusion by monocular occlusion. Type II (Franceschetti type) has no apparent underlying cause other than a debilitating illness or physical or emotional stress. Type III (Bielschowsky type) is associated with myopia [1-3]. The frequency of significant neurological diseases associated with esotropia is relatively low in patients with AACE [1-3]. However, recent studies recommend performing full neurological workups, including brain imaging, to rule out the possibility of a neurological disease in patients with AACE $[2,7]$.

After determining this, the treatment of diplopia will be considered. Horizontal diplopia caused by AACE significantly impairs patients' quality of life. Botox injections and prism glasses may be helpful for patients with small or moderate angle deviations of AACE. However, surgical treatment is usually necessary to achieve orthotropia and eliminate diplopia for patients with large-angle deviations of AACE, especially in patients without a significant neurological disease. Recently, the incidence of patients with AACE is increasing maybe due to excessive application of near vision linked to the display devices [6].

The surgical outcomes of AACE in previous studies are summarized in Table 3 [4,5,7-9]. Previous studies have shown that surgical outcomes are favorable in patients with AACE [4-9]. However, previous studies usually in-

Table 3. Previous studies reporting on surgical outcomes of patients with acute acquired concomitant esotropia

\begin{tabular}{|c|c|c|c|}
\hline Study & $\begin{array}{l}\text { Mean preoperative deviation } \\
\text { (distant / near) (PD) }\end{array}$ & $\begin{array}{l}\text { Surgical procedures } \\
\text { (no. of patients) }\end{array}$ & Summary of surgical outcome \\
\hline Sturm et al. (2011) [7] & $30.0 / 32.0$ & $\begin{array}{l}\text { Uni MR rec \& LR tucking (13) } \\
\text { Bi MR rec (6) } \\
\text { Bi MR rec\& LR tucking (6) }\end{array}$ & $\begin{array}{l}23 \mathrm{Pt}, \leq 8 \mathrm{PD} \\
3 \mathrm{Pt} \text {, additional surgery } \\
15 \mathrm{Pt} \text {, regained normal binocularity }\end{array}$ \\
\hline Chen et al. (2015) [5] & $40.7 / 41.0$ & $\begin{array}{l}\text { Uni MR rec \& LR res (16) } \\
\text { Uni or Bi MR rec (7) } \\
\text { Uni LR res (1) }\end{array}$ & $24 \mathrm{Pt}, \leq 10 \mathrm{PD}$, resolved diplopia \\
\hline Cai et al. (2019) [4] & $40.5 / 35.6$ & $\begin{array}{l}\text { Uni MR rec (4) } \\
\text { Bi MR rec (4) } \\
\text { Uni MR rec \& LR res (26) } \\
\text { Uni LR res (1) } \\
\text { Bi MR rec \& Uni LR res (9) }\end{array}$ & $44 \mathrm{Pt}$, resolved diplopia \\
\hline $\begin{array}{l}\text { Garcia-Basterra et al. } \\
\text { (2019) [8] }\end{array}$ & $23.0 / 22.7$ & Bi MR rec with adjustable suture (15) & $15 \mathrm{Pt}$, resolved diplopia \\
\hline $\begin{array}{l}\text { Lee and Kim } \\
\text { (2019) [9] }\end{array}$ & $27.9 / 28.6$ & Uni or Bi MR rec (16) & $\begin{array}{l}12 \mathrm{Pt} \leq 8 \mathrm{PD}, \text { no diplopia } \\
2 \mathrm{Pt} \text {, postoperative adjustment }\end{array}$ \\
\hline Current study & $40.0 / 40.0$ & Uni MR rec \& LR res (17) & $17 \mathrm{Pt}$, resolved diplopia \\
\hline
\end{tabular}

$\mathrm{Uni}=$ unilateral; $\mathrm{MR}=$ medial rectus; rec $=$ recession; $\mathrm{LR}=$ lateral rectus; $\mathrm{Pt}=$ patients; $\mathrm{PD}=$ prism diopters; $\mathrm{Bi}=$ bilateral; res $=$ resection. 
cluded patients that underwent different surgical methods or had moderate angles of preoperative esodeviation. The surgical outcomes of patients with large-angle deviations of AACE, including only the $\mathrm{R} \& \mathrm{R}$ procedure, have rarely been evaluated in the literature. Previous studies have not suggested a surgical dosage for the R\&R procedure in relation to large-angle deviations of AACE. Although the surgical dosage may be individualized according to the surgeon's preference and experience, we have attempted to propose surgical dosage guidelines for less experienced surgeons or those that only occasionally undertake strabismus surgery [10].

In the present study, all patients with AACE showed improvement in diplopia after surgical treatment. Most patients with decreasing stereoacuity showed improvement in stereoacuity after surgical treatment. No patients displayed over-correction or adduction limitations during the follow-up period. The unilateral R\&R procedure for patients with large-angle deviations of AACE showed favorable surgical outcomes. Our surgical dosage of unilateral $R \& R$ procedure for esodeviation was not significantly different from previous existing surgical dosage guidelines [11-13]. AACE may be different from common forms of esotropia, such as infantile and accommodative esotropia, or other types of restrictive or paretic strabismus. Patients with AACE displayed relatively favorable surgical outcomes compared with other types of esotropia. Therefore, surgical treatment may be considered as the primary treatment for patients with large-angle deviations of AACE. Recently, Chen et al. [5] suggested that AACE can be divided into 2 subgroups, those with relatively small-angle deviations and those with large-angle deviations ( $>20 \mathrm{PD}$ ) because the clinical characteristics and therapeutic approaches differ. We agree with their opinion regarding the classification of AACE based on the amount of ocular deviation. Such a classification appears to be appropriate considering the different therapeutic approaches for patients with AACE.

Lee and Kim [9] have recommended increasing the amount of MR recession to achieve better surgical outcomes in adult patients with AACE. Garcia-Basterra et al. [8] have also demonstrated that the average dose-response of bilateral MR recession was significantly less than that predicted using the standard guideline. An increased surgical dosage of MR recession may be needed to achieve favorable outcomes in patients with AACE.
We preferred to perform the R\&R procedure for patients with large-angle deviations of AACE for the following reasons. First, the effect of over-recession was minimized. A large amount of MR recession in both eyes may be required for the surgical treatment of patients with large-angle deviations of AACE. Small, inadvertent inaccuracies of large MR recessions $(>6-7 \mathrm{~mm})$ can cause dramatic changes in muscle force and result in unfavorable outcomes [14]. The relationship curve between muscle length and tension is not linear but exponential, as there is a precipitous loss of muscle force at the end of the curve when muscle slack is increased. Second, the mechanical force effect of the resected lateral rectus muscle may be stressed to prevent the recurrence of esodeviation [15]. The mechanical force caused by the resected lateral rectus muscle is postulated to enhance the effect of the MR recession. The resected muscle may be a means to ensure the effectiveness of the recession. Third, the contralateral un-operated eye can be saved in case a second surgery is required due to under-correction [11].

This study had some limitations. First, a comparison of surgical outcomes between the bilateral MR recession and the R\&R procedure for large-angle deviations of AACE was not performed. To clarify the surgical outcomes of $R \& R$ for large-angle deviations of AACE, a comparison of the surgical outcomes with bilateral MR recession may be needed. We do not usually perform bilateral MR recession for large-angle deviations of AACE. Instead, we prefer the $R \& R$ procedure due to experiencing under-correction with bilateral MR recessions. Second, this study included a relatively small number of patients with short operative follow-up period. Therefore, the investigation of associated clinical factors and surgical outcomes of long-term follow-up were not evaluated. For example, the surgical outcomes between adult and pediatric patients could not be compared. Future studies should consider these limitations.

\section{Conflict of Interest}

No potential conflict of interest relevant to this article was reported. 


\section{References}

1. Dotan G, Keshet Y, Qureshi HM, et al. When pediatric acute acquired comitant esotropia is not caused by a neurological disease. J AAPOS 2020;24:5.

2. Gilbert AL, Koo EB, Heidary G. Evaluation and management of acute acquired comitant esotropia in children. Semin Ophthalmol 2017;32:8-13.

3. Spierer A. Acute concomitant esotropia of adulthood. Ophthalmology 2003;110:1053-6.

4. Cai C, Dai H, Shen Y. Clinical characteristics and surgical outcomes of acute acquired Comitant Esotropia. BMC Ophthalmol 2019;19:173.

5. Chen J, Deng D, Sun Y, et al. Acute acquired concomitant esotropia: clinical features, classification, and etiology. Medicine (Baltimore) 2015;94:e2273.

6. Roda M, Pellegrini M, Rosti A, et al. Augmented bimedial rectus muscles recession in acute acquired concomitant esotropia associated with myopia. Can J Ophthalmol 2020:S0008-4182 (20)30776-6.

7. Sturm V, Menke MN, Knecht PB, Schoffler C. Long-term follow-up of children with acute acquired concomitant esotropia. J AAPOS 2011;15:317-20.

8. Garcia-Basterra I, Rodriguez Del Valle JM, Garcia-Ben A, et al. Outcomes of medial rectus recession with adjustable suture in acute concomitant esotropia of adulthood. J Pediatr Ophthalmol Strabismus 2019;56:101-6.

9. Lee HJ, Kim SJ. Clinical characteristics and surgical outcomes of adults with acute acquired comitant esotropia. Jpn J Ophthalmol 2019;63:483-9.

10. von Noorden GK, Campos EC. Principle of surgical treatment. In: von Noorden GK, Campos EC. Binocular vision and ocular motility. 6th ed. St. Louis: Mosby; 2002. p. 565631.

11. Agrawal S, Singh V, Gupta SK, Agrawal S. Evaluating a new surgical dosage calculation method for esotropia. Oman J Ophthalmol 2013;6:165-9.

12. Cestari DM, Hunter DG. Learning strabismus surgery: a case-based approach. Philadelphia: Wolter Kluwer; 2013. p. 231-2.

13. Wright K, Strube YN. Pediatric ophthalmology and strabismus. 3rd ed. New York: Oxford; 2012. p. 368-87.

14. Kushner BJ. Strabismus surgery. In: Kushner BJ. Strabismus. Gewerbestrasse: Springer; 2017. p. 153-92.

15. Kim WJ, Kim MM. The clinical course of recurrent intermittent exotropia following one or two surgeries over 24 months postoperatively. Eye (Lond) 2014;28:819-24. 